\section{ORIGINAL RESEARCH}

Y. Liu

S. Xiao

J. Liu

H. Zhou

Z. Liu

Y. Xin

W.Z. Suo

\title{
An Experimental Study of Acute Radiation- Induced Cognitive Dysfunction in a Young Rat Model
}

\begin{abstract}
BACKGROUND AND PURPOSE: Radiation-induced cognitive dysfunction is a common and serious clinical complication after radiation therapy for a brain tumor, but the knowledge of its mechanism is poorly understood. The purpose of this study was to establish a young rat model for acute radiationinduced cognitive dysfunction and associated BBB damage, as well as histopathologic changes.
\end{abstract}

MATERIALS AND METHODS: Young male rats were randomized into 4 groups to receive irradiation treatments at $300 \mathrm{cGy} / \mathrm{min}$ with doses of 0 (sham), 10, 20, and $40 \mathrm{~Gy}$, respectively. Each treatment group was further randomized into 4 subgroups for following up cognitive tests and assessment of their BBB integrity and potential histopathologic changes at $0,7,20$, and 60 days.

RESULTS: We found that irradiation at $10 \mathrm{~Gy}$ failed to induce any significant effects. Irradiation at 20 Gy resulted in a transient impairment of the cognitive functions at 7 and 20 days and returned to normal at 60 days. Irradiation at $40 \mathrm{~Gy}$ caused the severest cognitive impairment, which peaked at 7 days, and lasted for at least 60 days. The impaired cognition in both the 20-Gy and 40-Gy-irradiated rats was more or less accompanied with increased brain water content and deteriorated BBB function, though mild histopathologic alternations were only noticed in the 40-Gy-irradiated rats at 20 days.

CONCLUSION: A single-dose exposure at 20 to $40 \mathrm{~Gy}$ is sufficient to induce acute brain injury at both cognitive and pathologic levels in young male rats. In addition, morphologic outcomes may not be sensitive enough to reveal all of the pathologic changes, whereas BBB disruption may be an earlier and more sensitive index for acute RE. Therefore, the present model is useful for basic and therapeutic studies of acute RE.

ABBREVIATIONS: $\mathrm{BBB}=$ blood-brain barrier; $\mathrm{HE}=$ hematoxylin-eosin; $\mathrm{NPC}=$ nasopharyngeal carcinoma; $\mathrm{RE}=$ radiation encephalopathy; $\mathrm{s}=$ seconds; $\mathrm{EB}=$ Evans-Blue

$\mathbf{R}_{\mathrm{p}}^{\mathrm{a}}$ adiation therapy is used widely for the treatment of diffuse primary and metastatic brain tumors. ${ }^{1,2}$ As many as 200,000 patients receive partial large-field or whole-brain irradiation every year, and the population of long-term cancer survivors continues to grow. During treatment, however, some healthy brain tissues are also exposed to the radiation, and consequently many patients may experience symptoms associated with damage to these healthy tissues for a few weeks after therapy. Some of these symptoms may even last for months or years. This is known as acute and chronic RE. Especially because of neurocognitive impairment, RE is emerging as a major health problem. ${ }^{3}$

NPC in the Guangdong province of southeastern China constitutes approximately $32 \%$ of all cancers and is therefore

Received April 21, 2009; accepted after revision June 29.

From the Department of Neurology (Y.L., S.X., J.L., H.Z., Z.L., Y.X.), Second Affiliated Hospital, Sun Yat-sen University, Guangzhou, China; Department of Neurology (Y.L.), Tai'an City Central Hospital, Tai'an, China; Laboratory for Alzheimer's Disease and Aging Research (W.Z.S.), Veterans Affairs Medical Center, Kansas City, Missouri; and Departments of Neurology and Molecular and Integrative Physiology (W.Z.S.), University of Kansas Medical Center, Kansas City, Kansas.

Yunlin Liu and Songhua Xiao contributed equally to this work.

This study was supported by a grant to Jun Liu from the National Natural Science Foundation of China (No. 30870750).

Please address correspondence to Jun Liu, Department of Neurology, the Second Affiliated Hospital, Sun Yat-sen University, Guangdong, China 510120; e-mail:docliujun@hotmail.com

Indicates open access to non-subscribers at www.ajnr.org

DOI 10.3174/ajnr.A1801 the most common kind of cancer in the province. ${ }^{4}$ The incidence of overall NPC in the Guangdong province is more than 20 per 100,000 people every year among male patients. The rate in Cantonese speakers is double those in other dialect groups, such as the Hakka, Hokkien, and Chiu Chau ${ }^{5}$ and is, to the best of our knowledge, the highest in the world. ${ }^{6}$ For patients with NPC, radiation therapy is the first choice and sometimes is the only effective treatment. With the development of radiation therapy, the 5-year survival rate of patients with NPC is more than $50 \% .^{7}$ As the radiation fields cover both the face and neck regions and inevitably subject the bilateral inferior temporal lobes to radiation exposure, RE after radiation therapy for NPC was reported to occur in $0.9 \%$ to $4 \%{ }^{8}$ This includes a series of clinical symptoms, such as elevated intracranial pressure caused mainly by cerebral edema and the progressive deterioration of the hippocampalassociated learning and memory functions, ${ }^{9,10}$ which can be especially devastating to patients and caregivers.

Knowledge of the mechanisms underlying acute-onset RE after cranial irradiation is the basis for improving therapies and prophylaxes, but it is poorly understood. ${ }^{11,12}$ To better understand the pathogenesis of early changes after brain irradiation, it is very important to establish an animal model that allows detailed examinations of cognitive dysfunction along with the accompanying histopathologic changes.

In this study, we used a linear accelerator as the source of radiation to establish a young adult rat model for acute-onset radiation-induced brain injury and evaluated the changes in 
aspects of animal behavior, histopathology, and $\mathrm{BBB}$ permeability.

\section{Materials and Methods}

\section{Materials and Animals}

Animals. One hundred and sixty Sprague-Dawley rats (male, 4 weeks old, $110 \pm 20 \mathrm{~g}$ ) were randomized into 4 groups to receive irradiation treatments at $300 \mathrm{cGy} / \mathrm{min}$ with doses of 0 (sham), 10, 20, and $40 \mathrm{~Gy}$, respectively. The 40 rats in each treatment group were further randomized into 4 subgroups for follow-up cognitive tests, histopathologic examination, and evaluation of BBB permeability and brain water content at 4 different time points $(0,7,20$, and 60 days). The rats were housed socially in a temperature-controlled room with free access to food and water. Animal care and the experimental procedures in this study were approved by the Animal Care and Ethics Committee at the San Yat-sen University, China.

Irradiation. Rats were irradiated by use of gamma rays delivered by a linear accelerator (Primus Linear Accelerator; Siemens, Erlangen, Germany) at room temperature. Animals were anesthetized with pentobarbital (30 mg/kg, i.p.) and restrained in the sternal recumbent position on a treatment table. The head was centered in the exposure field with the eyes, neck, and body protected with customized lead shielding. For each group of the rats, a single dose of exposure ( 0,10 , 20 , and $40 \mathrm{~Gy}$, respectively) at a dose rate $300 \mathrm{cGy} / \mathrm{min}$ was given. The rats in the sham control group received 0 Gy of irradiation but underwent the same stress as did the others.

General observation and body weight. After the treatment, the rats were housed under identical experimental conditions to observe their feeding and drinking behavior, limb movement, as well as the local skin reaction to the irradiation. Their body weights were monitored weekly. All of these observations were recorded and used as indexes of potential changes after irradiation.

Morris water maze. The potential cognitive changes of the rats were evaluated by use of the Morris water maze test, which was performed chiefly according to the reported procedures. ${ }^{13} \mathrm{~A}$ circular water tank ( $120 \mathrm{~cm}$ in diameter, $40 \mathrm{~cm}$ in depth), filled with water at $26 \pm 2{ }^{\circ} \mathrm{C}$, was divided into 4 quadrants, with a platform $(10 \mathrm{~cm}$ in diameter), made of clear acryl acid resin, placed in the center of one of the 4 quadrants in the pool. A video camera and monitor over the pool were used for automatic video recording. The water maze tests began with visible training to teach the rats that there is a platform, and that it is the way to get out of the water. For this purpose, the animal was first placed on a platform, which was initially placed in the center of the pool and exposed $1 \mathrm{~cm}$ above the water surface, for $15 \mathrm{~s}$. Then, the animal was placed into the water and permitted to swim or search for the platform and climb up. The same procedure was repeated for 2 more trials, followed by trials with the platform switched to each of the quadrants. The experimental trials began with filling up the tank with more water and milk so that the platform was $1 \mathrm{~cm}$ below the surface of the water and was invisible for the rat. Five consecutive days of acquisition were performed, with each of the 4 trials per day beginning with the animal being placed into each of the 4 quadrants to initiate a 120 -s trial. For each trial, the rat was placed in one of the 4 quadrants; if the rat found the platform in 2 minutes, it was permitted to rest there for $30 \mathrm{~s}$ before finishing the training. If the rat failed to find the platform in 2 minutes, it was led to the platform for a rest of $30 \mathrm{~s}$. The latency of target finding and swimming distance (navigation test) were recorded.

In the last day of training, the platform was removed from the pool and the rat permitted to explore the tank for $60 \mathrm{~s}$. The percentage of swimming distance in the target quadrant to the total distance and the duration of stay in the target quadrant were recorded as the indexes of the test (spatial probing).

Immunohistochemical procedure. After the cognitive tests, 10 rats in each subgroup were further divided into 3 subgroups for histopathologic examination $(n=3)$, brain water content determination $(n=3)$, and BBB permeability assessment $(n=4)$, respectively. In addition, accidental death of 4 rats had reduced the sample number of the planned histopathologic examination for the subgroups of $20 \mathrm{~Gy}$ at 0 day and $40 \mathrm{~Gy}$ at 0,7 , and 20 days, respectively, from 3 to 2 rats. For histopathologic examination, rats were anesthetized with pentobarbital and perfused with prechilled $0.9 \% \mathrm{NaCl}$ solution to flush out the blood before they were fixed with $4 \%$ paraformaldehyde solution at $4^{\circ} \mathrm{C}$. The brains were dissected out and continually fixed in paraformaldehyde solution for 2 days before being embedded in paraffin. Tissue sections ( $6 \mu \mathrm{m}$ thick) were cut sequentially along a coronal profile and followed by standard HE procedures, as previously described. $^{14}$

Determination of brain water content. Three animals from each subgroup $(n=3)$ were subjected to brain water content determination by use of the dry-wet weight method. ${ }^{15}$ After decapitation, the whole brain was weighed immediately to obtain the wet weight. The tissue was then dried in an oven at $100^{\circ} \mathrm{C}$ and reweighed to obtain the dry weight. Brain water content was calculated as (wet weight-dry weight)/wet weight $\times 100 \%$.

BBB permeability assessment. Four animals from each subgroup $(n=4)$ were subjected to BBB permeability assessment. In brief, $2 \%$ EB dye $(3 \mathrm{~mL} / \mathrm{kg})$ was injected into the anesthetized rats through the femoral vein. A successful injection was indicated by the conjunctivae and limbs turning blue several seconds after the injection. The chest was then opened 1 hour later, and the brain was transcardially perfused with $300 \mathrm{~mL}$ of saline through the left ventricle until colorless perfusion fluid was obtained from the right atrium. After decapitation, the brains were obtained and weighed, followed by incubation in formamide solution at $50^{\circ} \mathrm{C}$ for 72 hours. The optical attenuation of the EB formamide solution was determined by spectrophotometry at $635 \mathrm{~nm}$, and BBB permeability was expressed as microgram of EB per milligram of brain tissue $(\mu \mathrm{g} / \mathrm{mg})$.

\section{Statistical Analysis}

All of the quantitative data were expressed as mean \pm SD and analyzed with SPSS 12.0 software (SPSS, Chicago, Illinois). Singleelement variance analysis (1-way ANOVA) was used for the behavioral experiment. The $t$ test (2-tailed) was used for other quantitative data. A value of $P<.05$ was considered statistically significant.

\section{Results}

Our postirradiation observation revealed that 8 of 40 rats in the 40 Gy group displayed mild local skin depilation, hyperemia, and edema for 8 to 15 days after the treatment. The rest of the rats in this group and all other groups were normal. In addition, all rats showed normal daily activities, including feeding and drinking. No paralysis and convulsion were observed, and body weight gain was no different among the groups.

Radiation Exposure Dose-Dependently Impairs Cognition A Morris water maze was used to assess the ability of place navigation and spatial probing for these rats. We found that 

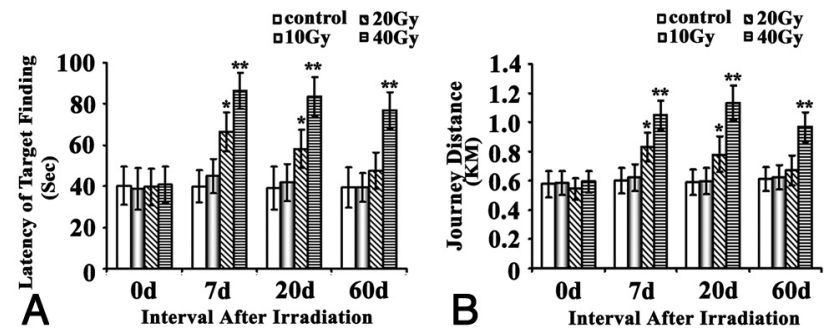

Fig 1. Effects of brain irradiation on place navigation function of rats in water maze. Rats exposed to different doses of irradiation treatments were subjected to the Morris water maze test. The latency of target finding $(A)$ and swimming distance $(B)$ was plotted and shown as indicated. ${ }^{*} P<.05,{ }^{* *} P<.01$, compared with their corresponding sham.
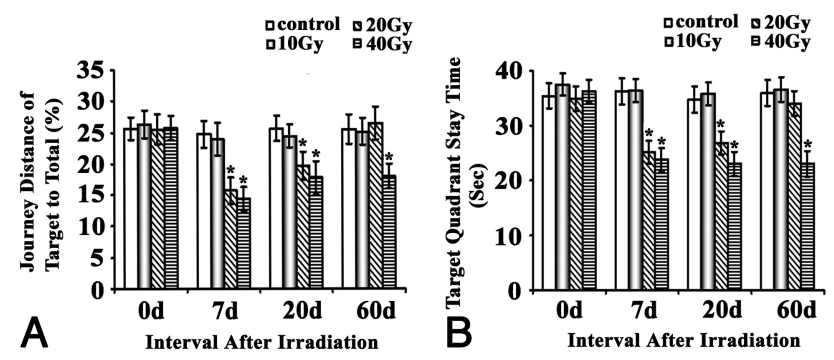

Fig 2. Effects of brain irradiation on space probe function of rats in the water maze. Rats exposed to different doses of irradiation treatments were subjected to the Morris water maze test. The journey distance of target to total $(A)$ and target quadrant staying time $(B)$ were plotted and shown as indicated. ${ }^{*} P<.05,{ }^{* *} P<.01$, compared with their corresponding sham.

the place navigational function of the rats was significantly impaired by the irradiation in a dose-dependent manner (Fig 1). In detail, 10-Gy irradiation failed to cause any impairment for the place navigation at any time points, whereas 20-Gy irradiation led to a transient impairment of the place navigation function as evident by their significantly increased latency $(P<.05)$ and swimming distance $(P<.05)$ compared with the sham control at 7 and 20 days, but not at 0 and 60 days. For $40-\mathrm{Gy}$ group, the impairment of place navigation function $(P<.01)$ was the severest among the 4 groups, as indicated by their longest latency and journey distance and the fact that the impairment lasted until at least 60 days. For all groups, there was no difference in the swimming speed.

Results for the spatial probe test were essentially in line with the place navigation results. Irradiation of $10 \mathrm{~Gy}$ did not cause any significant effects at any time points, 20 Gy resulted in a transient impairment at 7 and 20 days $(P<.05)$ but not at 60 days, and 40 Gy caused impairments that lasted until at least 60 days (Fig 2).

\section{Effect of Irradiation on Brain Water Content and BBB Permeability}

Examination of brain water content revealed no significant difference between the sham control group and the 10-Gy irradiation group at any time points (Fig 3). Irradiation at $20 \mathrm{~Gy}$ increased brain water content significantly $(P<.05) 7$ days after irradiation and this returned to normal when the content was examined at 20 and 60 days. Irradiation at $40 \mathrm{~Gy}$ induced the most increase in brain water content among all groups at all postirradiation time points $(P<.01)$ and lasted until at least 60 days. Changes in the BBB permeability caused by the irradiation were similar to the changes in the brain water con-
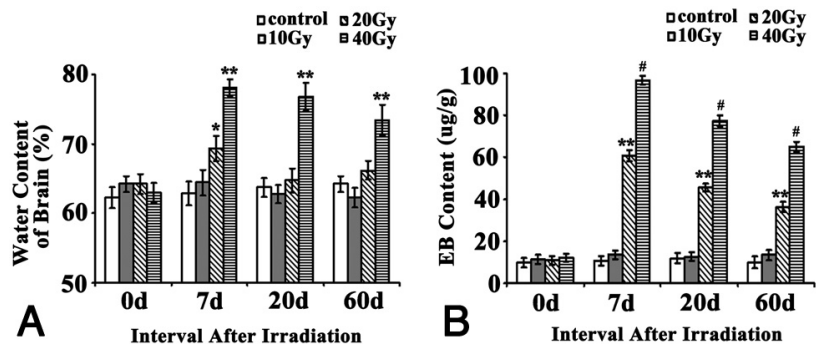

Fig 3. Effects of brain irradiation on brain water content and BBB permeability. $A$, Water content of brain, determined by the method of dry and wet weight. Water content in brain $=($ wet weight - dry weight $) /$ wet weight $\times 100 \%$. B, BBB permeability was expressed as micrograms of EB per milligrams of brain tissue $(\mu \mathrm{g} / \mathrm{g}) .{ }^{*} P<.05,{ }^{* *} P<$ .01 , \# $P<.001$, compared with their corresponding sham.

tent, only with minor differences: $10 \mathrm{~Gy}$ had no effects at all, but 20 Gy and 40 Gy both significantly $(P<.01$ and $P<.001$, respectively) impaired the $\mathrm{BBB}$ integrity and led to an increased BBB permeability from 7 days up to at least 60 days. Although both 20 Gy and 40 Gy impaired BBB integrity all along, the effects of $40 \mathrm{~Gy}$ were significantly stronger than that of $20 \mathrm{~Gy}$. In addition, although the changes induced by 20 and $40 \mathrm{~Gy}$ at all 3 postirradiation time points were significant, the peak change occurred at 7 days, with a trend of gradually recovering with the time, but still remaining far worse than normal even at 60 days.

\section{Pathologic Changes}

Conventional HE staining was used to assess the potential pathologic changes at the light microscopic level. We found no noticeable pathologic changes in the sham, 10-Gy, and 20-Gy irradiation groups at any examined time points. For the 40-Gy irradiation group, however, although no changes could be observed in the hippocampal region, some pathologic changes, such as partial loose and irregular arrangement of neurons and vacular degeneration, were found in the parietal white matter near the cortex at 20 days (Fig 4) but not at any other time points examined.

\section{Discussion}

Radiation therapy is routinely used in the treatment of intracranial, facial, and cervical tumors. However, radiation exposure to healthy brain tissues may cause several adverse effects such as RE. Acute RE, occurring a few days after irradiation at the end of radiation therapy, is a major health problem for patients who receive partial large-field or whole-brain irradiation. These patients often show a deterioration of almost all domains of memory, with a dose-dependent impairment in working memory. ${ }^{10}$ There is growing concern regarding the cognitive consequences of whole-brain irradiation for longterm survivors of cancer. ${ }^{16}$

Previous animal studies have addressed whole-brain irradiation-induced cognitive impairment after both single and fractionated radiation dose schedules by use of $\mathrm{x}$-ray and cobalt 60 as the source of radiation. ${ }^{17,18}$ As the linear accelerator becomes more and more widely applied, protocols with use of a linear accelerator as the source of radiation are getting more and more similar to conventional radiation therapy protocols used for patients in the clinic. In this study, we established an early-onset young rat model of radiation-induced cerebral in- 

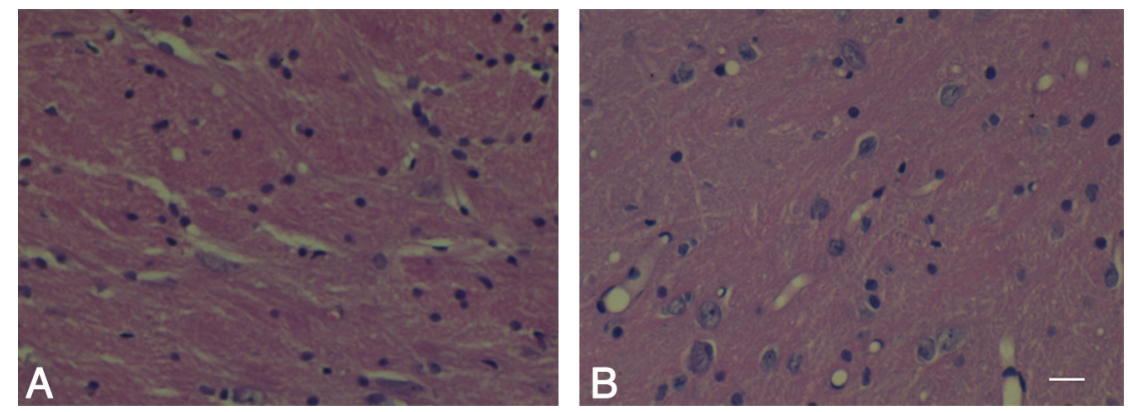

Fig 4. Pathologic change after irradiation. $A$ and $B$ represent the results of HE staining in the control group $(A)$ and the 40-Gy irradiated group 20 days after irradiation (B). It was noted that the partial loose and irregular arrangement of neurons and vacular degeneration in the parietal white matter near the cortex were found in the $40-\mathrm{Gy}$ irradiated group 20 days after irradiation. Scale bar, $40 \mu \mathrm{mol} / \mathrm{L}$.

jury that mimics the clinical manifestation to a large extent by testing different irradiation dosages (10,20, and $40 \mathrm{~Gy})$ with a linear accelerator.

Behavioral studies of the acute effect of cranial irradiation are relatively rare. To our knowledge, only a few reports have established cognitive dysfunction in rats exposed to irradiation but have mainly focused on late-onset RE. ${ }^{19}$ Hodges et $\mathrm{al}^{20}$ reported radiation-induced deficits in a $\mathrm{T}$-maze forced choice alteration and a subsequent dose-dependent water maze deficit during a period of 44 weeks. They indicated that local cranial irradiation with a low dose (20 Gy) of x-rays could produce a cognitive deficit in adult rats without evidence of pathologic changes. However, they could not find acute behavioral and neuropathologic effects. Lamproglou et $\mathrm{al}^{12}$ reported a radiation-induced (30 Gy) memory deficit at 1 month after irradiation in 4-month-old Wistar rats. They found that the response to the radiation observed in young rats differed from that observed in old rats. Young rats showed an earlier decrease in learning and memory than old rats, and this deficit was followed by partial recovery.

In our study, we used young rats ( 4 weeks) for the tests and found that the significant cognitive impairment could be detected 7 days after 20-Gy and 40-Gy irradiation. The deficit had lasted for at least 2 more weeks in the 20-Gy group and for an even longer duration (up to at least 60 days) with the higher-dose (40-Gy) irradiation. HE staining showed that mild histologic changes of brain edema could be observed in the higher-dose (40-Gy) group. This young rat model seems to be more valuable than the old rat model in the elucidation of the behavior and pathogenesis of cognitive dysfunction after irradiation because the young rat model displays more confounding changes at both the cognitive and pathologic levels and opens a longer time window for possible exploration of different therapeutic approaches.

Although the molecular mechanisms of RE after brain radiation remain to be elucidated, several proposals have been made, such as a reduction in regional glucose metabolism, ${ }^{21}$ induction of the production of inflammatory cytokines and mediators by microglia and astrocytes, ${ }^{22,23}$ increase of vascular permeability, etc. Shi et $\mathrm{al}^{24}$ found that the relative levels of certain subtypes of the $N$-methyl $D$-aspartate receptor were significantly higher in the hippocampal cornu ammonis 1 (CA1) region of irradiated rats compared with control rats after whole-brain radiation, indicating a possible involvement of an altered glutamate neurotransmission and/or excitatory neurotoxicity in radiation-induced cognitive impairments. Other mechanisms, such as the death of parenchymal and vascular cells, and even the inhibition of the formation of new neurons in the hippocampus, could also be involved. ${ }^{25}$ Given the fact that many proposed molecular mechanisms remain to be tested, the present acute model provides a means for further mechanistic studies for shorter experimental cycles.

Complications of irradiation may also arise as a consequence of vasogenic edema after disruption of the BBB. ${ }^{26}$ Disruption of the $\mathrm{BBB}$ is an early, readily recognizable pathophysiologic event occurring after radiation injury and is detectable in vivo and in vitro by MR imaging and histochemical studies. Several studies have reported the occurrence of a transient increase of BBB permeability after radiation exposure, though the duration seemed to vary among the reports, and it was suggested to strongly depend on the size and the physicochemical properties of the probes used for the assay. ${ }^{27}$ Nakata et $\mathrm{al}^{28}$ studied the effect of single irradiation on the permeability of the $\mathrm{BBB}$ in rat brains and found that the destruction of the $\mathrm{BBB}$ was detected as early as 1 day after irradiation with 20 or $40 \mathrm{~Gy}$, reached its maximum after 3 days, and gradually decreased during the following few weeks. The degree of this destruction could become much greater when the irradiation dose increased to $80 \mathrm{~Gy}$ and the $\mathrm{BBB}$ disruption remained during the whole course of the experiment.

Our study showed that irradiation at 20 Gy and 40 Gy increased the BBB permeability 7 days after the irradiation and lasted for the remaining experimental course. As the BBB is mainly composed of vascular endothelial cells, astroglial cells, and a basement membrane, other authors ${ }^{29}$ have indicated that dose-related changes in endothelial cell enlargement, vessel dilation, and basement membrane thickening, as well as the changes in vascular permeability and associated hypoxia could be observed after irradiation. In this study, the changes of BBB permeability were 4 -fold to 10 -fold greater than the level of sham, and such changes lasted almost through the entire experimental course after irradiation. In a cross-comparison of the nonbehavioral outcomes, the brain water content change was less than $20 \%$, whereas the histopathologic change was mild. Therefore, the change in BBB permeability seems to be the most sensitive and reliable index for the detection of radiation brain injury from early radiation, and this, along with the cognitive tests, should provide essential assessment for the rat model and help us develop new therapies and prophylaxis for RE. 


\section{Conclusions}

Our study demonstrated that radiation-induced brain injury in rats is dose dependent, and 20 to $40 \mathrm{~Gy}$ of a single-dose exposure is sufficient to induce acute brain injury at both the cognitive and pathologic levels in young rats. In addition, morphologic outcomes may not be sensitive enough to reveal all of the pathologic changes, whereas the BBB disruption may be an earlier and more sensitive index for acute RE. Therefore, the present model is useful for basic and therapeutic studies of acute RE.

\section{References}

1. Tsao MN, Lloyd NS, Wong RK, et al. Radiotherapeutic management of brain metastases: a systematic review and meta-analysis. Cancer Treat Rev 2005;31:256-73

2. Kantor G, Laprie A, Huchet A, et al. Radiation therapy for glial tumors: technical aspects and clinical indications. Cancer Radiother 2008;12:687-94

3. Coleman CN, Stone HB, Moulder JE, et al. Modulation of radiation injury. Science 2004;304:693-94

4. Yan L, Xi Z, Drettner B. Epidemiological studies of nasopharyngeal cancer in the Guangzhou area, China preliminary report. Acta Otolaryngol 1989;107:424-27

5. Li CC, Yu MC, Henderson BE. Some epidemiologic observations of nasopharyngeal carcinoma in Guangdong, People's Republic of China. Natl Cancer Inst Monogr 1985;69:49-52

6. Chang ET, Adami HO. The enigmatic epidemiology of nasopharyngeal carcinoma. Cancer Epidemiol Biomarkers Prev 2006;15:1765-77

7. Langleben DD, Segall GM. PET in differentiation of recurrent brain tumor from radiation injury. $J$ Nucl Med 2000;41:1861-67

8. Chong VF, Fan YF, Mukherji SK. Radiation-induced temporal lobe changes: CT and MR imaging characteristics. AJR Am J Roentgenol 2000;175:431-36

9. Roman DD, Sperduto PW. Neuropsychological effects of cranial radiation: current knowledge and future directions. Int J Radiat Oncol Biol Phys 1995;31:983-98

10. Welzel G, Fleckenstein K, Mai SK, et al. Acute neurocognitive impairment during cranial radiation therapy in patients with intracranial tumors. Strahlenther Onkol 2008;184:647-54

11. Crossen JR, Garwood D, Glatstein E, et al. Neurobehavioral sequelae of cranial irradiation in adults: a review of radiation-induced encephalopathy. J Clin Oncol 1994;12:627-42

12. Lamproglou I, Chen QM, Boisserie G, et al. Radiation-induced cognitive dysfunction: an experimental model in the old rat. Int J Radiat Oncol Biol Phys 1995;31:65-70
13. Morris RG, Garrud P, Rawlins JN, et al. Place navigation impaired in rats with hippocampal lesions. Nature 1982;297:681-83

14. Suo Z, Fang C, Crawford F, et al. Superoxide free radical and intracellular calcium mediate A beta(1-42) induced endothelial toxicity. Brain Res 1997;762:144-52

15. Whalen MJ, Carlos TM, Wisniewski SR, et al. Effect of neutropenia and granulocyte colony stimulating factor-induced neutrophilia on blood-brain barrier permeability and brain edema after traumatic brain injury in rats. Crit Care Med 2000;28:3710-17

16. Johannesen $\mathrm{TB}$, Lien $\mathrm{HH}$, Hole $\mathrm{KH}$, et al. Radiological and clinical assessment of long-term brain tumour survivors after radiotherapy. Radiother Oncol 2003;69:169-76

17. Serduc R, van de Looij Y, Francony G, et al. Characterization and quantification of cerebral edema induced by synchrotron $x$-ray microbeam radiation therapy. Phys Med Biol 2008;53:1153-66

18. Kobierska A, Karnicka-Młodkowska H, Solska E. [Radiotherapy of neoplasms of the central nervous system.] Neurol Neurochir Pol 1983;17:85-90

19. Yoneoka Y, Satoh M, Akiyama K, et al. An experimental study of radiationinduced cognitive dysfunction in an adult rat model. $\mathrm{Br} \mathrm{J}$ Radiol 1999;72:1196-201

20. Hodges H, Katzung N, Sowinski P, et al. Late behavioural and neuropathological effects of local brain irradiation in the rat. Behav Brain Res 1998;91:99-114

21. Delattre JY, Shapiro WR, Ponsner JB. Acute effects of low-dose cranial irradiation on regional capillary permeability in experimental brain tumors. $\mathrm{J} \mathrm{Neu}$ rol Sci 1989;90:147-53

22. Chiang CS, McBride WH. Radiation enhances tumor necrosis factor alpha production by murine brain cells. Brain Res 1991;566:265-69

23. Hayakawa K, Borchardt PE, Sakuma S, et al. Microglial cytokine gene induction after irradiation is affected by morphologic differentiation. Radiat Med 1997; 15:405-10

24. Shi L, Adams MM, Long A. Spatial learning and memory deficits after wholebrain irradiation are associated with changes in NMDA receptor subunits in the hippocampus. Radiation Res 2006;166:892-99

25. Madsen TM, Kristjansen PE, Bolwig TG, et al. Arrested neuronal proliferation and impaired hippocampal function following fractionated irradiation in the adult rat. Neuroscience 2003;119:635-42

26. St George EJ, Perks J, Plowman PN. Stereotactic radiosurgery XIV: The role of the haemosiderin 'ring' in the development of adverse reactions following radiosurgery for intracranial cavernous malformations: a sustainable hypothesis. Br J Neurosurg 2002;16:385-91

27. Diserbo M, Agin A, Lamproglou I, et al. Blood-brain barrier permeability after gamma whole-body irradiation: an in vivo microdialysis study. Can J Physiol Pharmacol 2002;80:670-78

28. Nakata H, Yoshimine T, Murasawa A, et al. Early blood-brain barrier disruption after high-dose single-fraction irradiation in rats. Acta Neurochir (Wien) 1995; 136:82-86

29. Hopewell JW, Calvo W, Campling D. Effects of radiation on the microvasculature. Implications for normal-tissue damage. Front Radiat Ther Oncol 1989: 23:85-95 